Digilec 8 (2021), pp. 164-179

Fecha de recepción: 26/07/2021

Fecha de aceptación: 14/09/2021

DOI: https://doi.org/10.17979/digilec.2021.8.0.8639

(c)

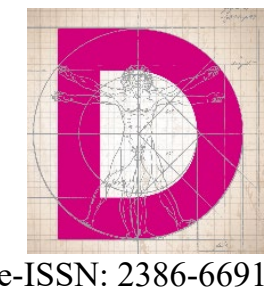

\title{
LOS INTERVALOS MUSICALES EN EL ENTORNO SONORO DEL ESTUDIANTADO: PROPUESTA PARA LA REALIZACIÓN DE UN JUEGO DE ESCAPISMO EDUCATIVO
}

\section{MUSICAL INTERVALS IN THE STUDENTS' SOUND CONTEXT: A PROPOSAL FOR THE DEVELOPMENT OF AN EDUCATIONAL BREAKOUT GAME}

\author{
Jesús TENORIO DEL RÍO \\ Universidad Internacional de Valencia-VIU \\ Orcid: https://orcid.org/0000-0002-6599-4056 \\ Noelia LEITE COSQUE* \\ Universidade da Coruña \\ Orcid: https://orcid.org/0000-0003-1180-4920
}

\begin{abstract}
Resumen
Uno de los retos presentes en el aula de música reside en la comprensión y asimilación de los intervalos musicales. Esta propuesta, dirigida especialmente al alumnado de educación secundaria, busca presentar una herramienta orientada a la asociación del intervalo musical con elementos presentes en el entorno del estudiantado. Con este planteamiento se pretende mejorar su comprensión, retención y asociación de una forma significativa, ligando los contenidos a elementos presentes en su mundo sonoro. Para ello es necesario llevar a cabo un entrenamiento auditivo gracias al cual se desarrolle este contenido. Aprovechando dichas fuentes acústicas, escuchadas de manera habitual y que se encuentran claramente codificadas en sus procesos mentales, se puede optimizar el tiempo dedicado a su estudio. Además, la gamificación incide positivamente en la predisposición del alumnado a afrontar nuevos retos en su formación. De las múltiples ventanas que abre esta metodología, los recientemente surgidos juegos de escapismo educativo se postulan como una de las herramientas más apropiadas para un aprendizaje vivencial. Es por ello que, además de la explicación de qué es un intervalo musical y de cuáles son sus especies, se propondrá un concurso en el que se buscarán ejemplos de los mismos en la música que consumen habitualmente. Posteriormente, se reforzará su desempeño de forma auditiva, visual y teórica por medio del juego de escapismo.
\end{abstract}

* Facultade de Ciencias da Educación. Campus de Elviña, s/n. 15071 A Coruña. Email: susotenorio@gmail.com,noelia.leite@udc.es 
Palabras clave: música; intervalos; aprendizaje significativo; gamificación; juego de escapismo

\begin{abstract}
One of the challenges present in the music classroom lies in the understanding and assimilation of musical intervals. This proposal, aimed especially at secondary school pupils, seeks to present a tool designed for the association of musical intervals with elements present in the students' environment. Therefore, this approach focuses on enhancing their understanding, retention and association significantly, linking these contents to other components present in the students' surroundings. In order to do this, it is necessary to carry out an auditory training in which this content is developed. The use of these acoustic sources, which are listened to regularly and clearly encoded in their mental processes, optimizes the time spent on their study. In addition, gamification has a positive impact on the willingness of students to face new challenges in their education. Within the windows of opportunity that this methodology offers, the educational breakout games, recently developed, are one of the most appropriate tools for experiential learning. That is why, apart from the explanation of music intervals and their species, students will be required to look for examples of intervals in the music they usually consume as part of a contest. Subsequently, their performance will be reinforced in an auditory, visual and theoretical way through the breakout game.
\end{abstract}

Key Words: music; intervals; meaningful learning; gamification; breakout game 


\section{INTRODUCCIÓN}

La presente propuesta busca el desarrollo de una actividad que permita el aprendizaje de los intervalos musicales desde un punto de vista práctico y teórico. Está dirigida al alumnado de educación secundaria -aunque es perfectamente adaptable a las enseñanzas musicales- con el fin de relacionar dicho concepto con el contexto sonoro que le rodea añadiendo un componente lúdico.

Se tomarán como punto de partida los antecedentes pedagógicos de los denominados métodos activos (Hemsy de Gainza, 2004), concretamente con las figuras de Edgar Willems y de E. Jaques-Dalcroze y su concepto holístico del contexto del alumnado y el aprendizaje musical dentro del aula. Asimismo, se hará uso de la metodología de la gamificación, en aras de ofrecer una actividad que facilite el aprendizaje significativo usando como referencia la dinámica de los juegos de escapismo.

\subsection{Base pedagógica}

Las metodologías musicales activas surgidas en el siglo XX pretenden desarrollar la creatividad, la imaginación y la participación del alumnado apostando por una educación musical vivencial y activa (Brufal Arráez, 2013).

Dentro de los métodos activos se encuentran los planteamientos pedagógicos de Dalcroze y Willems (Hemsy de Gainza, 2004). En ellos se aprecian relaciones entre las experiencias educativas y la percepción sensorial, mediante el entorno sonoro en el que se desarrolla el alumnado (Cuevas Romero, 2015). El primero utiliza el espacio como parte del fenómeno sonoro y motor, siendo intermediario de la comunicación (Vernia Carrasco, 2012). El segundo parte de los principios vitales del ser humano -voz y movimiento- (Porcel Carreño, 2010), siendo característico el uso de materiales sonoros variados como instrumentos u objetos cotidianos (Brufal Arráez, 2013). Siguiendo a Willems (2001: 51), "mediante la educación podemos despertar, dirigir, desarrollar el funcionamiento del órgano del oído y eso tiene tanta importancia que, si se le deja por su cuenta, el oído corre el riesgo de atrofiarse".

Dentro de los fundamentos de las metodologías musicales activas se encuentra, precisamente, su ya comentado componente vivencial. Así, se genera un aprendizaje basado en la experimentación. Esta forma de aprender se relaciona con el aprendizaje significativo, en el que se asocian nuevos conocimientos con otros adquiridos previamente. Tal y como plantea Ausubel (2002), este proceso asociativo se lleva a cabo haciendo uso de materiales que guardan relación con algún aspecto o contenido ya asimilado -imágenes, símbolos o conceptos- o con ideas no tan concretas pero apropiadas. Según el propio autor, el aprendizaje significativo tiene dos requisitos principales (102-106): 
- "Actitud de aprendizaje significativa" por parte del alumnado: búsqueda de comprensión de los conceptos empleando términos y conexiones abstractas que faciliten la asimilación.

- Uso de "materiales potencialmente significativos": fácilmente enlazables con otros conocimientos de una forma "no arbitraria y no literal", lo cual depende del tipo de actividad que se realice y de las características concretas de la persona que aprende en cuanto a conocimientos previos, coeficiente intelectual, edad y contexto sociocultural.

Este tipo de aprendizaje, por tanto, facilita una mejor y más duradera asimilación de nuevos contenidos. A su vez, incide positivamente en la comprensión de estos otorgando un papel activo en el estudiantado al requerir de este que seleccione, analice y compare aquellos elementos ya presentes que se relacionan con los nuevos. Finalmente, también le exige la realización de una reordenación de toda la información, tanto nueva como ya existente, lo cual dependerá de las cualidades de la persona (Ausubel, 2002).

\subsection{Recursos metodológicos}

La enseñanza a través del juego se puede encontrar desde pensadores como Platón, que propone que el proceso de aprendizaje se realice desde el juego (Ballén Molina, 2010), y en la actualidad es una de las tendencias dentro de las aulas. En este apartado se hará una contextualización de las diferentes técnicas utilizadas para este fin y que serán las empleadas en esta propuesta.

\subsubsection{Gamificación}

En 2011 Pelling acuñó el término gamification, adaptado al español mediante el anglicismo gamificación, refiriéndose a la posibilidad de utilizar elementos propios de los juegos en tareas de la vida real (Renobell Santaren y García Gaiteiro, 2016). Esta será la metodología principal en la que se basará esta propuesta. Según Salazar et al. (2016), esta estrategia educativa se fundamenta en la utilización de dinámicas de juego que generen la participación del alumnado y que, por medio de su estructura lúdica, permitan adquirir y desarrollar conceptos y competencias.

Siguiendo a Kapp (2012), se pueden observar diferentes elementos dentro de la definición de gamificación: que esté basado en las dinámicas de un juego, ayudando a los/las participantes a invertir tiempo en la actividad; las mecánicas, incluyendo los niveles, las puntuaciones, etc., para dinamizar la experiencia; la estética, que propicie una inmersión en el juego; el pensamiento de juego, por medio de elementos de competitividad, cooperación o narrativos; el compromiso de los propios jugadores y jugadoras, ya sean consumidores, consumidoras o estudiantes; la motivación, para lo cual se debe adecuar el nivel, sin que sea demasiado complicado ni sencillo; el fomento del aprendizaje, puesto que este es uno de los objetivos de la gamificación; y, la resolución de problemas, dado que esta estrategia educativa tiene un gran potencial para suplir algunas de las dificultades surgidas habitualmente durante el proceso de enseñanza- 
aprendizaje. Teixes (2014) ratifica este último punto, ejemplificándolo con la dificultad de concentración, el nivel de conocimientos previos y la influencia tanto de un mal ambiente de estudio, como de factores emocionales o la falta de motivación.

\subsubsection{Breakout educativo}

Uno de los modelos de gamificación integrado recientemente en el ámbito educativo es el conocido como breakout-o escape room que literalmente consiste en salir de un lugar-. Nació como una actividad de entretenimiento alrededor de 2007 en Japón, pero ha ganado popularidad alrededor de todo el mundo (García Lázaro, 2019).

Este juego tiene como objetivo principal lograr salir de un espacio resolviendo diferentes desafíos, que se superarán mediante pistas, rompecabezas o tareas. Wiemker et al. (2016) establecen tres categorías dentro de los juegos de escapismo según la secuenciación de los retos. Estos son el modelo lineal, en el que dicha secuencia está predeterminada; el modelo abierto en el que se pueden resolver los desafíos en el orden que prefieran los participantes y el multilineal, el cual combina ambas categorías. Asimismo, se pueden basar tanto en la modalidad competitiva, en la que se juega contra otro equipo en espacios idénticos, en los puntos, obtenidos tras realizar las tareas o de larga escala, sin tiempo límite, siendo el objetivo salir en el menor tiempo posible (Wiemker et al., 2016).

Tal y como señala García Lázaro (2019), es conveniente que todo breakout educativo siga ciertas recomendaciones, entre las que se destacan la adecuación del nivel de dificultad del juego a las características concretas del grupo al que va dirigido y, el control de los tiempos con el fin de evitar la tediosidad y consecuente pérdida de atención. Por último, siempre es interesante conocer las impresiones de los jugadores y de las jugadoras, con vistas a mejorar y enriquecer el juego de cara al futuro.

La integración de este tipo de actividades en el aula permite -gracias al amplio abanico de escenarios en el que se puede ambientar- desarrollar destrezas y habilidades tales como la resolución de problemas y el pensamiento crítico, así como también el trabajo en equipo cuando se realicen de forma colaborativa (Wiemker et al., 2016).

\subsubsection{Uso de las TIC}

Los juegos de escapismo se pueden llevar a cabo de forma física o virtual. La presente propuesta se diseñará para el segundo formato, de tal forma que permita desarrollar destrezas en el ámbito de las Tecnologías de la Información y la Comunicación.

Los continuos avances tecnológicos y la generalización del acceso a internet son una constante en la actual Era Digital, tal y como exponen Viñals Blanco y Cuenca Amigo (2016). Desde un punto de vista social, esto ha supuesto cambios en todos los ámbitos, incluido el educativo. Además, estos autores califican esta sociedad digital como cambiante, compleja, caótica y ubicua. En lo referente al ámbito educativo, destacan la relevancia que adquiere el aprendizaje informal al ser más adecuado para este contexto. 
Mañas Pérez y Roig-Vila (2019), concretan el concepto de TIC en tres aspectos que son la informática, las telecomunicaciones y las tecnologías audiovisuales. Siguiendo a estos autores, estas herramientas educativas, especialmente en el caso de internet, permiten en base al aprendizaje significativo desarrollar habilidades cognitivas e interactivas. Además, también posibilitan la superación de limitaciones a la educación a nivel personal, geográfico y socioeconómico.

Todas estas cuestiones influyen en las metodologías y también en los roles docente y discente. Tal y como plantean Viñals Blanco y Cuenca Amigo (2016: 109), "en la Era Digital la manera de aprender ha cambiado $\mathrm{y}$, por ende, la forma de enseñar debe adaptarse". En cuanto a la figura docente, sus actuales funciones serán guiar, organizar y acompañar a sus discentes en el proceso de enseñanza-aprendizaje. Este rol cobra una mayor importancia en cuanto al diseño, elaboración y adaptación de los materiales y recursos educativos de los que dispondrá el alumnado (Mañas Pérez y Roig-Vila, 2019). En consecuencia, el estudiantado pasa a tener un papel más activo y autodidacta. Además, cabe destacar que estos son los "nativos digitales", denominados así por Marc Prensky (2001), los cuales se caracterizan por presentar unas habilidades innatas para las tecnologías, estar acostumbrados a la inmediatez y a tener predilección por una formación lúdica.

\subsection{Los intervalos musicales}

La premisa de este trabajo será conseguir el aprendizaje de los intervalos musicales relacionando los mismos con el contexto sonoro del estudiantado. Para ello, es necesario en primer lugar obtener una definición de los mismos. Un intervalo musical es la distancia -refiriéndose a la diferencia de altura- entre dos notas, habitualmente expresada en tonos y semitonos (Roca y Molina, 2006). En función del orden entre ambas notas pueden ser ascendentes o descendentes. Atendiendo a la distancia pueden ser conjuntos o disjuntos. Según su simultaneidad, pueden ser armónicos o melódicos. Por último, por el rango, serán simples si no superan la octava, o compuestos a partir de los intervalos de novena (Mayor Ibáñez y De Pedro Cursá, 2009).

Se pueden clasificar de diferentes formas, siguiendo distintos parámetros. Según su número de tonos y de semitonos, estos pueden ser disminuidos, menores, mayores y aumentados en el caso de los intervalos de segunda, tercera, sexta y séptima, mientras que en los de cuarta, quinta y octava serán disminuidos, justos y aumentados. Entre las diferentes clasificaciones hay un semitono de diferencia, siendo los disminuidos los más pequeños (Roca y Molina, 2006).

\section{OBJETIVOS}

El diseño de esta propuesta tiene como foco la elaboración de una actividad en la que el alumnado participante consiga identificar los intervalos musicales -distancia entre dos notas-, así como las distintas especies de los mismos, aunando la vertiente práctica y la teórica para adquirir un aprendizaje holístico. En base a esto, el objetivo principal será 
el de diseñar una intervención que facilite el aprendizaje significativo de los intervalos musicales. Para tal fin, se han establecido tres objetivos complementarios que serán:

- Facilitar el reconocimiento auditivo y visual de los intervalos musicales.

- Asociar el concepto de intervalo musical al contexto sonoro del alumnado.

- Adquirir habilidades en referencia al empleo de las TIC.

\section{METODOLOGÍA}

Los fundamentos de Willems y Dalcroze ya comentados, así como los recursos metodológicos referentes a la gamificación, sirven de base para el planteamiento de esta propuesta. En ella, se tratará de relacionar los contenidos, concretamente los intervalos musicales, con el contexto sonoro en el que se desenvuelve el alumnado.

Como ya se ha comentado, esta actividad se puede llevar a cabo tanto desde la materia de Música en educación secundaria, como desde el aula de Lenguaje Musical dentro de las enseñanzas de música, bien sea en el conservatorio o en las escuelas de música. Para este caso, se ejemplificará esta propuesta para el aula de Música de secundaria, en la cual cada sesión tendrá una duración de 50 minutos.

Esta iniciativa se planteará para ser desarrollada en dos sesiones. Durante la primera clase tendrá lugar una exposición participativa de los conceptos teóricos, con vistas a que el alumnado pueda ampliar sus nociones de lenguaje musical y una segunda parte más lúdica, con dinámica de concurso. La segunda sesión, se dividirá en tres partes: repaso de conceptos, juego de escapismo y puesta en común.

\subsection{Secuenciación de las sesiones}

Para poder abordar los contenidos, es necesaria una explicación previa por parte del profesorado. Se hará uso, acorde al nivel evolutivo del estudiantado, del pensamiento abstracto, ya que permite mejorar la eficacia de la asimilación de nuevos conceptos; yendo de lo general a lo específico (Ausubel, 2002).

\subsubsection{Primera sesión}

La actividad comenzará con una exposición participativa en la que el rol docente consistirá en exponer al estudiantado las nociones teóricas necesarias para el desarrollo posterior del juego, explicando qué es un intervalo musical, cómo calcular la distancia entre dos notas musicales y presentando las distintas especies que se pueden encontrar. En este momento de la actividad es importante que se escuche al alumnado, resolviendo sus dudas e intercambiando impresiones, para lograr así que la totalidad del grupo, independientemente de sus capacidades, destrezas y conocimientos previos, entienda los contenidos; de tal forma que, posteriormente, puedan deducir los tipos de intervalo a los que se enfrentarán.

Conviene matizar que, para no dar lugar a equívocos, es necesario realizar una clara diferenciación de la unidad de medida -tonos y semitonos-y la distancia entre dos notas. 
Es por ello que se debe afianzar cómo calcular el intervalo entre notas, contado en tonos y semitonos, para poder realizar la clasificación en disminuidos, menores, mayores, justos y aumentados. Este concepto no debe interferir en lo referente a la clasificación numérica de los intervalos de las notas, siendo el foco de la confusión la distancia entre notas segunda, tercera, etc.- con el número en tonos y semitonos - un tono, dos tonos, etc.--

El agrupamiento para esta primera sesión será, al inicio, del conjunto del aula y, posteriormente, se harán grupos cooperativos de tres o cuatro estudiantes. En estos, se mezclarán discentes con más destrezas en la materia con quienes necesiten más apoyo para lograr que el aprendizaje se realice de forma conjunta, ayudándose entre sí.

La segunda actividad de esta sesión tendrá como fin el reconocimiento auditivo de los intervalos. Para ello, se hará uso de canciones que incluyan -ya sea al inicio de su introducción o de su estribillo- el tipo de intervalo requerido, con ejemplos actualizados y del gusto del alumnado; fortaleciendo así la vinculación del estudiantado con la materia. Esta conexión es tan beneficiosa como necesaria, tal y como advierten Oriola Requena y Gustems Carnicer (2015). Posteriormente, se realizará un concurso en el que cada grupo debe aportar nuevos ejemplos, provenientes de sus preferencias musicales. Será, por tanto, un juego en equipo, con el objetivo de aportar el mayor número de ejemplos correctos, ayudándose de la competitividad, mencionada por Kapp (2012), para favorecer el aprendizaje. De esta forma se introduce la gamificación, haciendo que la clase entre en un ambiente lúdico a la par que didáctico, mientras se afianza tanto el reconocimiento auditivo, como la capacidad de entonar dichos intervalos.

El diseño de esta sesión sigue lo planteado hace ya más de una década por Hemsy de Gainza (2010). Según esta autora, conviene centrarse en el hecho musical desde una posición activa, que haga uso de la variedad de repertorio existente y que fomente la creatividad. De esta forma, se seguirá el proceder de otras áreas del conocimiento, en las que se prioriza la práctica, dando lugar a un aprendizaje integrado en permanente contacto con la realidad.

\subsubsection{Segunda sesión}

Se comenzará con un repaso de los conocimientos aprendidos en la sesión anterior. Durante el mismo, el profesorado intervendrá lo menos posible dejando espacio al alumnado para hacer un repaso colectivo.

En la segunda parte de la sesión se realizará la actividad principal de esta propuesta, el juego de escapismo. Este complementará los conceptos ya aprendidos por medio del aprendizaje significativo, asociando los intervalos -estudiados de forma teórica e introducidos auditivamente- al contexto sonoro del estudiantado desde un enfoque práctico, participativo y autónomo. Por último, se realizará una puesta en común, intercambiando opiniones e impresiones sobre el juego, su nivel de dificultad, las sensaciones experimentadas durante su realización, el nivel de conocimientos adquiridos, la utilidad de la propuesta, etc.

El juego estará planteado para ser completado en un período de 20 minutos, prorrogable hasta los 30 minutos con el fin de que todo el alumnado consiga completarlo. 
Dicha limitación temporal se relaciona con la naturaleza de los juegos lúdicos de breakout, a la vez que genera tensión y competitividad -sana- entre los participantes. Además, será beneficioso de cara a mantener la atención del alumnado a lo largo de la actividad.

Al igual que en la primera sesión, la actividad inicial se realizará de forma colectiva. Para el juego de escapismo, se mantendrán los grupos cooperativos de 3 o 4 personas de la sesión anterior. Finalmente, se hará un intercambio colectivo de opiniones e impresiones sobre el juego y una encuesta a nivel individual para analizar la experiencia.

\subsection{Juego}

Para el desarrollo del juego de escapismo se ha de tener en cuenta, en primer lugar, el mundo sonoro que rodea al estudiantado, el cual incluye todo tipo de fuentes acústicas. A continuación, y con la intención de integrar los contenidos a desarrollar, se seleccionarán los distintos elementos acústicos apropiados para el estudio de los intervalos musicales presentes en dicho entorno. De esta forma se llevará a cabo un entrenamiento auditivo, el cual facilitará la asimilación de los contenidos teóricos a través del aprendizaje significativo. Además de esta preparación auditiva, se trabajarán aspectos teóricos y de reconocimiento visual de los intervalos.

El juego se estructurará en cuatro secciones, dedicando cada una de ellas a una de las especies de intervalos: menores, mayores, justos y aumentados/disminuidos. Estas dos últimas categorías se harán de forma conjunta, relacionándolas con los intervalos anteriores y buscando solamente el conocimiento teórico y el reconocimiento visual.

Para superar cada una de las cuatro secciones, se plantearán tres tipos de preguntas. Estas serán teóricas, de reconocimiento visual o de reconocimiento auditivo. Al final de cada una de las secciones, como es usual en este tipo de juegos se entregará una pista, que en este caso será un número. Será imprescindible obtener los cuatro dígitos ordenados para finalizar la actividad. Es recomendable que el número resultante tenga un significado, el cual el alumnado tendrá que investigar por su propia cuenta. Este número estará relacionado con algún aspecto de la música -ya sea compositivo, histórico, etc.de modo que este juego pueda ser también una oportunidad de apertura hacia otros contenidos presentes en el currículum de la asignatura.

\section{MATERIALES}

Para la realización de esta propuesta será necesaria la preparación de ciertos materiales y recursos. Estos serán de creación propia en base a la teoría musical, aportando un nuevo enfoque al aprendizaje de los intervalos musicales.

\subsection{Aprendizaje de los intervalos musicales}

Durante la primera sesión, en la cual se comienza la explicación de los conceptos teóricos referentes a los intervalos musicales, se utilizará la escala de Do Mayor como 
base para los ejemplos. Esta decisión viene dada por la necesidad de simplificar al alumnado el cálculo de distancias, pues al carecer de alteraciones propias, se evita parte de la confusión al contar los tonos y los semitonos entre las notas.

En un primer momento, se explicará qué es un intervalo, según la definición anteriormente citada. A continuación, se expondrá su clasificación según su amplitud diatónica -especificación numérica-y las distintas especies. Se finalizará la explicación con el reconocimiento visual y auditivo.

Para la segunda actividad de la sesión se utilizarán melodías presentes en la música actual, concretamente, de la popular entre el alumnado. Aunque idealmente es preferible conocer previamente los gustos musicales presentes en el grupo aula, en esta ejemplificación se utilizarán canciones provenientes de la lista "Canciones más escuchadas: España" de Spotify (2021).

- $4^{\mathrm{a}}$ justa ascendente: “KESI", Camilo (introducción).

- $5^{\mathrm{a}}$ justa ascendente $+3^{\mathrm{a}}$ menor descendente: "Juramento eterno de sal", Álvaro De Luna (introducción).

- $7^{a}$ mayor ascendente: "Pareja del año", Sebastian Yatra y Myke Towers (estribillo).

Cabe destacar que es recomendable prestar atención a las letras de las canciones utilizadas como ejemplo, pues es habitual que en la música popular actual se traten de forma inadecuada cuestiones relativas a las relaciones interpersonales, el trato hacia la mujer, la educación sexual, hábitos de vida saludables, etc. Por ello, sería interesante invitar al alumnado a la reflexión en este sentido en el caso de que alguna de las canciones propuestas presente esta problemática, sin que por ello deje de ser un ejemplo válido o puntuable para el concurso.

\subsection{Breakout de intervalos musicales}

El eje principal de esta propuesta, como ya se ha mencionado, es el juego de escapismo educativo. Este se realizará en un soporte informático, de forma que ayude a desarrollar también las competencias TIC en el alumnado.

En este tipo de juegos hay, habitualmente, una historia que actúa como hilo conductor entre las distintas fases, a la par que ayuda a la inmersión del jugador o de la jugadora. En este caso, y con vistas a fomentar un aprendizaje vivencial, se narrará una situación cotidiana con la que el estudiantado pueda identificarse. Además, es precisamente esta historia la que sitúa el contexto sonoro, ya que los escenarios o elementos utilizados en esta ofrecerán las fuentes sonoras.

\subsubsection{Descripción}

El soporte técnico del juego se hará a través de un programa que permita la creación de diapositivas. Habrá una inicial a modo de portada, que dará acceso a la que narra la historia previa al inicio de la partida. En esta propuesta, se hará uso del siguiente hilo argumental: 
Un/Una integrante de vuestra pandilla se muda hoy a otro país. Es un día de lluvia, pero aun asi decidís hacerle una despedida sorpresa en su casa. Al llegar allí, esta se encuentra ya vacía. Intentáis localizarlo/a llamando a su nuevo número, el cual había anotado en una de vuestras agendas. Aqui empieza vuestra aventura: la mochila se habia mojado, haciendo que los cuatro últimos digitos se encuentren ilegibles.

MISIÓN: Tenéis que completar el número de teléfono e intentar llegar a tiempo al aeropuerto para despediros. ;Las puertas de embarque se cierran en 20 minutos!

Como se puede observar, en la propia historia se establece el contexto, la misión a realizar y el tiempo del que se dispone para conseguir superar todas las fases. Es importante utilizar un lenguaje directo con un vocabulario asequible para el nivel del alumnado, de forma que la realización de las tareas no se vea condicionada por dificultades de comprensión, pues el objetivo principal sigue siendo el aprendizaje de los intervalos musicales.

Habrá una única misión, que será la de completar el juego antes de que finalicen los 20 minutos - prorrogables a 30 para que el total de la clase consiga completarlo-. Para superar el juego con éxito, se deben conseguir los cuatro dígitos. Cada uno de ellos será dado al finalizar cada una de las cuatro fases en las que se divide el juego.

\subsubsection{Secciones}

Cada una de las secciones estará ambientada en un escenario diferente. En cada una de estas salas se encontrarán ejemplos de las distintas especies de intervalos a trabajar. A continuación, se ejemplificarán las salas, indicando el espacio, la especie y el elemento sonoro propuesto para el reconocimiento auditivo:

Sección 1. Casa - Intervalos menores. Timbre de la entrada. Comúnmente este sonido utiliza una tercera menor descendente.

Sección 2. Aeropuerto - Intervalos mayores. Tono de aviso de la megafonía, arpegio mayor ascendente. El alumnado deberá tener en cuenta las dos primeras notas para obtener una tercera mayor.

Sección 3. Ascensor - Intervalos justos. Sonido del ascensor, habitualmente formado por un arpegio triada mayor descendente. Teniendo en cuenta la primera y la última nota, se obtiene una quinta justa.

Sección 4. Punto de control - Intervalos disminuidos y aumentados. No se busca su reconocimiento auditivo, sino crear una relación entre intervalos menores, mayores y justos con disminuidos y aumentados. Habrá dos preguntas teóricas y una de reconocimiento visual.

Cada una de dichas secciones en las que se divide el juego estará integrada por tres preguntas tipo test, con tres opciones de respuesta, de las cuales solo una es correcta. Estas preguntas serán de tres tipos: teoría, reconocimiento visual y reconocimiento auditivo. 
Por último, para este ejemplo los cuatro dígitos obtenidos al final de cada sección serán los propios de la serie de Fibonacci $(2,3,5,8)$, lo que podría ser un punto de partida hacia el estudio del serialismo.

\subsubsection{Requisitos}

Esta experiencia se realizará a través de un programa informático que permita el diseño de las diapositivas, las cuales conducirán el juego. Este software puede ser incluso una plataforma en internet. Algunos puntos a tener en cuenta son que se permita el uso de texto, imagen y sonido en una misma ventana, así como la posibilidad de seleccionar respuestas correctas o incorrectas. Siguiendo esto, debe poder enlazarse cada respuesta con la pantalla correspondiente, ya sea para avanzar en el juego o advirtiendo de que se ha cometido un error.

Además de los recursos habituales para el desarrollo de la actividad lectiva en el aula, para esta propuesta será necesario disponer de un ordenador con altavoces o auriculares para cada uno de los grupos de estudiantes. Cabe destacar que en función del programa o plataforma que se utilice, puede ser también necesaria la conexión a internet.

\section{CONCLUSIONES}

Llegados a este punto, conviene analizar la propuesta en cuanto a la consecución de objetivos y su adecuación, así como a la coherencia con el marco teórico planteado al inicio. Por otro lado, se mostrarán los posibles beneficios que esta intervención puede aportar, así como la adaptabilidad y la prospectiva de este trabajo.

\subsection{Análisis de la propuesta}

Durante la realización de esta propuesta se ha podido cumplir el principal objetivo, que es diseñar una intervención que facilite el aprendizaje significativo de los intervalos musicales. Esto es posible gracias a la relación creada entre los nuevos conocimientos con otros previamente asimilados por el alumnado. Por medio del concurso, se integra la música que consume habitualmente el alumnado con los contenidos de la asignatura, mientras que por medio del juego de escapismo, se relacionan los intervalos con el contexto sonoro en el que se desenvuelven.

Asimismo, también se facilita el reconocimiento auditivo y visual de los intervalos, al ser trabajados mediante un entorno de diversión, en lugar de las formas convencionales, habitualmente tediosas. Además, de esta forma, el aprendizaje es vivencial, pues el alumnado adquiere un rol activo, al desarrollar los contenidos de forma teórica y, sobre todo, práctica, fomentando la deducción desde un primer momento.

Se logra también asociar el concepto de intervalo al contexto sonoro del alumnado mediante el juego de escapismo, en el que los ejemplos son sonidos que están presentes habitualmente en espacios tanto públicos como privados. 
Por último, en cuanto a la consecución de los objetivos complementarios, el hecho de que el juego se realice en un soporte informático ayuda a que el alumnado adquiera habilidades en referencia al empleo de las TIC, familiarizándose con su uso desde un punto de vista no solo lúdico, sino también educativo. Aunque esta competencia no se desarrolla especialmente desde la materia de Música, se debe atender a la transversalidad, sobre todo de cara a la sociedad actual. Esta, exige tener destrezas en el manejo de las TIC, independientemente del ámbito en el que una persona pueda relacionarse -laboral, administrativo, educativo, etc.-. La integración de estas herramientas en el aula, sin que ello suponga reemplazar o abandonar otras técnicas analógicas, enriquece la experiencia educativa y ofrece una formación más completa al alumnado.

Dado que esta propuesta se basa en la gamificación es necesario que cumpla ciertos requisitos, como se ha reflejado en el marco teórico de la misma. Estos seguirán lo planteado por Kapp (2012), empleando la mecánica y dinámicas de un juego -fases, estética, competitividad y cooperación- y fomentando el aprendizaje; adaptando el nivel de la propuesta al alumnado al que va dirigida. Además, teniendo en cuenta la ya mencionada vivencialidad, se espera que se sienten las bases para que se produzca el aprendizaje, que además, será previsiblemente significativo, dado que cumple los requisitos propuestos por Ausubel (2002) para que esto suceda.

Asimismo, cumple también los desafíos propios de los juegos de escapismo recopilados por Wiemker et al. (2016) y García Lázaro (2019), usando la modalidad lineal y competitiva, con tiempo límite. Sigue así dichas recomendaciones, que son principalmente la adecuación de la dificultad, el control de los tiempos y la posibilidad de recopilar las impresiones de los/las participantes tras la partida.

Los beneficios que puede traer consigo la implementación de esta propuesta serán la mejora en la asimilación de los contenidos, al aunar los dos mundos sonoros -aula de música y preferencias musicales- en los que vive el alumnado; la mejora de la escucha y su relación con el reconocimiento visual y teórico de los intervalos; y el desarrollo del trabajo en grupo, fomentando el compañerismo y el esfuerzo, aun basándose en la competitividad entre equipos.

\subsection{Adaptabilidad y prospectiva de la propuesta}

Tal y como se ha destacado a lo largo de esta investigación, esta propuesta da pie a multitud de adaptaciones, tanto temáticas, como narrativas, de adecuación al nivel o de su puesta en marcha en diferentes soportes. De esta forma, se podrá variar tanto la historia que sirve como hilo conductor del juego a la actualidad o a las inquietudes del alumnado, como los diferentes contextos sonoros que vendrán dados, precisamente, por las distintas temáticas.

Son destacables las posibilidades de adaptabilidad de cara a llevar a cabo el juego de escapismo en la realidad. Utilizando los espacios del centro educativo o de un teatro se puede generar un aprendizaje aún más vivencial. Las principales limitaciones dependerán de la cantidad de recursos -e incluso tiempo de preparación, en caso de ser una instalación ajena- de los que se dispongan. 
Además, es posible la adecuación a diferentes niveles de dificultad, tanto superior como inferior, modificando las tareas a realizar durante el progreso del juego. Por último y, como ya se ha comentado anteriormente, el código de cuatro dígitos puede servir como nexo de unión a diferentes temas; ya sea utilizando un año destacable en la historia de la música, secuencias armónicas, relación con títulos de obras, etc.

En cuanto a la prospectiva de la propuesta, esta puede ser tomada o bien como punto de partida para trabajos de mayor envergadura, o bien para realizar otros materiales educativos con el mismo planteamiento teórico.

Dado que esta intervención no se ha podido poner en práctica todavía, sería adecuado comprobar los planteamientos reflejados en este artículo. Además, para obtener una mayor profundidad en los resultados, sería conveniente que se pudieran comparar las conclusiones obtenidas en dos grupos de control, uno en el que se realice por medio de las actividades planteadas y otro en el que se haga de las formas tradicionales. Así, se podrá comprobar si esta metodología marca una diferencia real en el aprendizaje del alumnado.

Por otro lado, también se podrían utilizar los planteamientos e ideas presentadas con otros conceptos tanto musicales como de otras asignaturas, para tratar de desarrollar y utilizar nuevas herramientas metodológicas.

\section{REFERENCIAS BIBLIOGRÁFICAS}

Ausubel, D. P. (2002). Adquisición y retención del conocimiento. Una perspectiva cognitiva, G. Sánchez Barberán (Trad.). Paidós.

Ballén Molina, R. (2010). La pedagogía en los diálogos de Platón. Diálogos de saberes: $\begin{array}{llll}\text { investigaciones } \quad y \quad \text { ciencias } & \text { sociales, } 33,54 .\end{array}$ https://dialnet.unirioja.es/servlet/articulo? codigo=3618399

Brufal Arráez, J. D. (2013). Los principales métodos activos de educación musical en primaria: diferentes enfoques, particularidades y directrices básicas para el trabajo en el aula. Revista ArtsEduca, 5, 6-21. https://dialnet.unirioja.es/servlet/articulo? codigo $=4339750$

Cuevas Romero, S. (2015). La trascendencia de la educación musical de principios del siglo xx en la enseñanza actual. MAGISTER Revista de Formación del Profesorado e Investigación Educativa, 37-43. https://doi.org/10.1016/j.magis.2015.05.002

García Lázaro, I. (2019). Breakout como propuesta de gamificación en educación. $\begin{array}{llll}\text { HEKADEMOS Revista Educativa Digital, 27, } & \text { 71-79. }\end{array}$ https://hekademos.com/index.php/hekademos/article/view/17

Hemsy De Gainza, V. (2004). La educación musical en el siglo XX. Revista Musical Chilena, 58(201), 74-81. http://dx.doi.org/10.4067/S0716-27902004020100004

Hemsy De Gainza, V. (2010). Temas y problemáticas de la educación musical en la actualidad. AULA Revista de Pedagogía de la Universidad de Salamanca, 16, 3348. https://dialnet.unirioja.es/servlet/articulo? codigo $=3325931$ 
Kapp, K. M. (2012). The gamification of learning and instruction: game-based methods and strategies for training and education. San Francisco: Pfeiffer.

Mañas Pérez, A. y Roig-Vila, R. (2019). Las Tecnologías de la Información y la Comunicación en el ámbito educativo. Un tándem necesario en el contexto de la sociedad actual. Revista Internacional d'Humanitats, 45, 75-86. http://hdl.handle.net/10045/82089

Mayor Ibáñez, A. y De Pedro Cursá, D. (2009). Nuevos Cuadernos de Teoría. Grado Elemental 2. Real Musical.

Oriola Requena, S. y Gustems Carnicer, J. (2015). Música y adolescencia: usos, funciones y consideraciones educativas. UT Revista de Ciències de l'Educació (2), 28-45. https://doi.org/10.17345/ute.2015.2.660

Porcel Carreño, A. M. (2010). Metodologías musicales del S. XX. Aplicación en el aula. Revista Digital Innovación y Experiencias Educativas, 37, 1-10. https://archivos.csif.es/archivos/andalucia/ensenanza/revistas/csicsif/revista/pdf/N umero_37/ANA_MARIA_PORCEL_2.pdf

Prensky, M. (2001). Digital Natives, Digital Immigrants. On the Horizon (MCB University Press), 9(5), 1-6. https://marcprensky.com/writing/Prensky\%20\%20Digital\%20Natives, \%20Digital\%20Immigrants\%20-\%20Part1.pdf

Renobell Santaren, V. y García Gaiteiro, F. (2016). Gamificación en la educación: Reinventando la rueda. Revista DIM: Didáctica, Innovación y Multimedia, 34, 4558. http://dimglobal.net/revistaDIM34/docs/DIMAP34gamificacion.pdf

Roca, D. y Molina, E. (2006). Vademecum musical. Definición y ejemplos de términos. Metodología IEM. Madrid: Enclave Creativa.

Salazar Romero, A. D. C., Beltrán Flandoli, A. M. y Loaiza Aguirre, M. I. (2016). Proyecto Ascendere: Un ecosistema de prácticas de Innovación Docente en la UTPL. En In-Red 2016. II Congreso nacional de innovación educativa y docencia en red, V. Botti Navarro y M. A. Fernández Prada (Eds.), 1465-1480. Valencia: Universitat Politècnica de València. https://doi.org/10.4995/INRED2016.2016.4299

Spotify (2021). Canciones más escuchadas: España. Spotify. https://open.spotify.com/playlist/37i9dQZEVXbJwoKy8qKpHG?si=470bc094158 $54 \mathrm{a} 9 \mathrm{~b}$

Teixes, F. (2014). Gamificación: fundamentos y aplicaciones. UOC.

Vernia Carrasco, A. M. (2012). Método pedagógico musical Dalcroze. ARTSEDUCA Revista electrónica de educación en las artes, 1, 24-27. http://hdl.handle.net/10234/152468

Viñals Blanco, A. y Cuenca Amigo, J. (2016). El rol docente en la era digital. Revista Interuniversitaria de Formación del Profesorado 30(2), 103-114. https://www.redalyc.org/pdf/274/27447325008.pdf

Wiemker, M., Elumir, E., y Clare, A. (2015). Breakout Games: Can you transform an unpleasant situation into a pleasant one?. En Game Based Learning Dialogorientierung \& Spielerisches Lernen Digital und Analog, J. Haag, J. Weißenböck, W. Gruber y C. F. Freisleben-Teutscher (Eds.), 55-68. Viena: St. 
Pölten University of Applied Sciences. http://skill.fhstp.ac.at/wpcontent/uploads/2016/05/Tagungsband 2015.pdf

Willems, E. (2001). El oído musical. La preparación auditiva del niño, M. C. Medina (Trad.). Barcelona: Paidós. 